Bol. Acad. peru. leng. 67. 2020 (179-203)

\title{
LA ENSEÑANZA DE LA LENGUA INDÍGENA Y EL DESARROLLO DE LA INTERCULTURALIDAD EN LAS HORAS DE CLASES DE LENGUA NATIVA COMO L1 Y L2 EN EL PERÚ
}

\section{THE TEACHING OF THE INDIGENOUS LANGUAGE AND THE DEVELOPMENT OF INTERCULTURALITY DURING NATIVE LANGUAGE CLASSES SUCH AS L1 AND L2 IN PERU}

\author{
Marco Antonio Lovón Cueva \\ Universidad Nacional Mayor de San Marcos \\ mlovonc@unmsm.edu.pe \\ https://orcid.org/0000-0002-9182-6072 \\ Danny Loysi Chávez Sánchez \\ Universidad Nacional Mayor de San Marcos \\ zoejadeshoko@gmail.com \\ https://orcid.org/0000-0001-9182-6710 \\ Edgar Mauro Yalta Gonzales \\ Universidad Nacional Mayor de San Marcos \\ edgar.yalta@unmsm.edu.pe \\ https:/orcid.org/0000-0001-8444-6779
}


https://doi.org/10.46744/bapl.202001.006

\author{
Ana María García Liza \\ Universidad Nacional Mayor de San Marcos \\ agarcial@unmsm.edu.pe \\ https://orcid.org/0000-0003-0505-1299
}

\title{
Resumen:
}

La educación bilingüe intercultural (EBI) en el Perú no es homogénea. Las situaciones de aprendizaje y enseñanza de las lenguas indígenas son diferentes, así como la aplicación del concepto de la interculturalidad. Las lenguas originarias pueden encontrarse en estado de primera o de segunda lengua. El objetivo de esta investigación es analizar dos situaciones de EBI en el Perú. Para ello, se revisa las realidades del shipibo y del asháninka. Metodológicamente, se describen y comparan los contextos educativos. El trabajo concluye con priorizar la enseñanza y aprendizaje según las prioridades de cada situación sociolingüística.

Palabras clave: educación, bilingüismo, interculturalidad, shipibo, asháninka.

\section{Abstract:}

Intercultural bilingual education (IBE) in Peru is not homogenous. The learning and teaching situations of indigenous languages are different, as is the application of the concept of interculturality. Native languages can be found in a first or second language state. The purpose of this research is to analyze two IBE situations in Peru. To this end, the Shipibo and Ashaninka realities are reviewed. Methodologically, this paper describes and compares their educational contexts. The work concludes with an emphasis on teaching and learning according to the priorities of each sociolinguistic situation.

Key words: education, bilingualism, interculturality, Shipibo, Ashaninka.

Fecha de recepción:

Fecha de aceptación:
$10 / 02 / 2020$

$25 / 04 / 2020$ 
En el caso peruano, su realidad multilingüe, multicultural y diglósica constituye el contexto dentro del cual una numerosa población realiza su aprendizaje. El papel que juegan y la cultura en el proceso educativo reviste una importancia decisiva y, en razón de ello, resulta necesario impulsar un tratamiento adecuado de las lenguas y de la cultura en la educación, bajo la modalidad de la Educación Bilingüe Intercultural (EBI) (Godenzzi, 2001).

\section{Introducción}

Cuando se piensa en la EBI de los pueblos indígenas peruanos, se ha manifestado en diferentes ocasiones que todo es una declaración de buenas intenciones. Estamos lejos de la EBI en la práctica, todavía es un terreno de lo demagógico. Nosotros creemos que es la única opción educativa para los niños y niñas de los pueblos indígenas y, por ello, nos oponemos a que se siga diciendo qué y cómo se va trabajar en las aulas, pues no se han diversificado los currículos, no se cuenta con maestros capacitados y, lo que es peor, los propios docentes no saben qué significa ser un maestro EBI (Vigil, 2004: 4).

De acuerdo con la cita, la falta de la EBI se encuentra en su aplicación, y quizá también en la manera de concebir la situación EBI para los diversos pueblos, pues el sistema educativo peruano, por ejemplo, no contempla que haya comunidades indígenas en donde la primera lengua (L1) ya no es la lengua indígena, sino que esta es la segunda lengua (L2) y el castellano, la materna.

Hasta la fecha, la EBI en el Perú se ha basado en un solo tipo de experiencia y en una sola de las situaciones sociolingüísticas existentes en el país: la educación diseñada para niños y niñas monolingües de lengua indígena que deben aprender el castellano como L2 (López y Küper, 2002; Zuñiga y Ansión, 1997, 2002; Zúñiga, 2008). Si bien esta situación persiste en muchas localidades del país, es necesario también tomar en cuenta 
que el monolingüismo en lengua indígena resulta cada vez más escaso, dado el avance del bilingüismo (en el que el castellano predomina) de los educandos peruanos como resultado de los distintos procesos socioculturales y económicos en los que se ven inmersos (Vigil, 2004: 5).

En el Perú, la interculturalidad como principio rector del sistema educativo no es entendida, asimilada ni trabajada en las aulas, la cual debe propiciar la valoración y construcción de la identidad personal y cultural y la interrelación con otros individuos y colectividades.

Desarrollar las áreas del currículo desde un enfoque intercultural no ha pasado de ser un bonito enunciado hasta el momento. Muchos docentes se preguntan cómo hacer esto, y sobre todo, qué significa abordar las competencias y capacidades del currículo de manera intercultural (Burga, 2003: 12).

$\mathrm{Al}$ reconocer como valederas las posturas anteriores, interesa observar el tratamiento de la interculturalidad y la aplicación de la lengua indígena en las horas de enseñanza y aprendizaje. Para ello, estudiamos dos tipos de realidades sociolingüísticas diferentes: la del shipibo-konibo en estado de L1 y la del asháninka en estado de L2. Nos preguntamos: ¿Cuál es la situación de la enseñanza y aprendizaje del shipibo de Canantipishca como L1 y del asháninka del Perené como L2 en las horas de clases de lengua nativa? y ¿cómo se incorpora el concepto y práctica de la interculturalidad? El asháninka y el shipibo son dos lenguas amazónicas mayoritarias, sin embargo, el número de hablantes en cifras invisibiliza la situación particular de cada zona de hablantes. Por eso, hemos decidido optar por dos realidades específicas.

\section{Lineamientos generales}

\subsection{EBI para todos}

La EBI es un proceso y una actividad continua que propicia la construcción y valoración de la propia identidad, personal y cultural, como base para relacionarse con otros individuos y colectividades. Con ella, se 
https://doi.org/10.46744/bapl.202001.006

apunta hacia una sociedad culturalmente diversa y un mundo cada vez más interdependiente, pues asume que no existe una sola manera de ver el mundo y que uno mismo puede desarrollar nuevos conocimientos y actitudes al conocer otras formas de vida.

La educación intercultural permite desarrollar competencias en múltiples sistemas de percepción, pensamiento y acción, así como aprender a desenvolverse en diversos entornos culturales y a aprovechar sus aportes y contenidos. En ese sentido, constituyen un requisito fundamental para el logro de un pensamiento crítico y pluralista (Waish, 2000: 6).

Con un modelo EBI efectivo, se desarrollan capacidades cognitivas de desarrollo por encima de las diferencias. Sin embargo, se sabe que la EBI en el Perú está descontextualizada, pues no toma en consideración la situación real y expectativas de los pueblos indígenas.

Se concibe una EBI en la que solo se contemplan los casos donde la lengua indígena es la L1 y el castellano es la L2. No se tiene presente que, debido a los distintos procesos socioculturales y económicos en los que se ven inmersos los educandos de los pueblos indígenas, el monolingüismo en la lengua indígena resulta cada vez más escaso. No se toman en cuenta los casos en los que los pueblos indígenas han perdido la lengua indígena y quieren, en un proceso de reafirmación cultural, reaprenderla y piden que esta sea enseñada con metodología de segunda lengua (Vigil, 2004: 2).

Sabemos, sin embargo, que la realidad sociolingüística es diferente y que el grupo cuya L1 es la lengua indígena no es precisamente el caso más frecuente. Ese era el Perú descrito por Inés Pozzi Escott (sic) hace treinta años. Desde esa fecha, el Perú ha cambiado mucho y el avance del castellano ha sido grande. Hay distintas situaciones de uso de lenguas. Hay una castellanización galopante que no es sano negar. Es necesario pensar en distintas propuestas de EBI que repercutan positivamente en el mantenimiento de las lenguas indígenas (Vigil, 2005: 13).

Así lo planteaba en su momento la DINEBI: 
https://doi.org/10.46744/bapl.202001.006

Nuestra realidad multilingüe genera diversas situaciones de bilingüismo y de contacto de lenguas, las cuales constituyen el ámbito en el que se realiza la tarea educativa. La educación debe tomar en consideración la diversidad de las lenguas y las variedades que se dan en cada una de ellas, y dales un tratamiento adecuado, es decir, debe apuntar al desarrollo y conocimiento de la lengua materna (o de uso predominante), sea esta indígena o castellana, al aprendizaje de una segunda lengua, sea esta castellano o lengua indígena, $\mathrm{y}$ al aprendizaje de al menos una lengua extranjera (se respeta la cursiva original). (Ministerio de Educación-DINEBI, 2002: 4)

Sin embargo, muchas veces parece olvidarse que la EBI involucra no solo el tratamiento y respeto por las lenguas, sino también por la cultura general. En este contexto, se debe comenzar con el respeto y la valoración de la propia cultura, así como de las que están a nuestro alrededor, y empoderar a las menos favorecidas para colocarlas en una situación de igualdad. La interculturalidad promueve que los individuos fortalezcan su identidad.

\subsection{La lengua indígena como lengua materna y segunda lengua}

El papel que cumplen las lenguas en el proceso educativo reviste una importancia decisiva y, debido a ello, resulta necesario superar las diferencias en la enseñanza de lenguas indígenas y en castellano para que la EBI pueda convertirse en la modalidad adecuada para atender poblaciones, hasta hace muy poco, postergadas por la política educativa peruana (Vigil, 2004: 1).

Las lenguas indígenas del Perú pueden encontrarse en dos estados: primeras lenguas o segundas lenguas (Lovón, 2016). Esto debido a que son o no son habladas por la generación de niños que habitan en las comunidades de habla de una lengua autóctona. Muchos sabemos la importancia que manifiestan en relación con la lengua materna, ya que «la lengua materna constituye el vínculo social e individual por excelencia, la cual permite que cada uno se asuma como miembro de un grupo social específico. En la primera infancia, es portadora esencial del desarrollo psíquico, emocional, social y cognitivo» (Ministerio de Educación-DINEBI, 2002: 7). 
Por esto, el Estado se plantea mejorar y fortalecer las capacidades comunicativas orales como uno de sus objetivos, el cual puede ser realizado mediante el desarrollo de la producción escrita.

En el 2002, DINEBI señalaba lo siguiente:

Especificidades de la enseñanza de la lengua indígena como L1.

Objetivos específicos

1. Fortalecer en los educandos el uso de la lengua indígena, tanto en la comunicación interpersonal como en las actividades cognitivas complejas a partir de los contenidos de su cotidianeidad, acordes con su visión del mundo para su desempeño autónomo y crítico en el mundo del conocimiento y del trabajo.

2. Mejorar en los educandos su capacidad de comunicación en la lengua indígena materna a través del desarrollo de la lectura, la escritura, la reflexión metalingüística y la producción escrita.

3. Normar, planificar y conducir acciones educativas eficaces para la literacidad.

4. Contribuir en los procesos de estandarización de la escritura de las lenguas indígenas. (Ministerio de Educación-DINEBI, 2002: 6)

Por otro lado, hay lenguas indígenas como L2 y que la EBI estimula su aprendizaje.

En las comunidades indígenas que han perdido o están en peligro de perder su lengua originaria, o esta ha sido desplazada por el castellano, la educación bilingüe estimula el aprendizaje de estas lenguas como una segunda lengua (Ministerio de Educación-DINEBI, 2002, 12).

El descubrimiento de la diversidad como riqueza y la progresiva toma de conciencia sobre los derechos lingüísticos culturales, conduce a que algunas comunidades y pueblos, que están en riesgo de perder su lengua indígena ancestral, hagan esfuerzos por revitalizarla mediante la exigencia de aprenderla como segunda lengua, ya que la lengua de uso predominante para ellos ya es el castellano (Ministerio de Educación-DINEBI, 2002: 7). 
https://doi.org/10.46744/bapl.202001.006

De igual manera, se plantean objetivos en relación con la enseñanza de la lengua indígena como segunda lengua.

Enseñanza de una lengua indígena como L2.

Objetivos específicos

1. Promover la valoración de las lenguas indígenas y así evitar su extinción.

2. Iniciar el proceso de recuperación de la lengua indígena en aquellas comunidades en al que los educando ya no la hablan.

3. Propiciar que lo educandos no indígenas aprendan una lengua originaria como segunda lengua.

4. Ganar espacios para el uso de las lenguas indígenas. (Ministerio de Educación-DINEBI, 2002: 7)

\subsection{El concepto de la interculturalidad}

La interculturalidad está referida explícitamente a la dimensión cultural del proceso educativo y al aprendizaje social y cultural que busca las necesidades básicas de los educandos (Trapnell, 2009; Trapnell, Calderón y Flores, 2008). «La interculturalidad es la situación de respeto, tolerancia, intercambio y diálogo productivo entre los diferentes grupos étnicos culturales, en el que cada uno aporta a los otros. Es la solución al problema de choque de cultura, a la pérdida de identidad, marginación y olvido» (Heise, 2001: 13). Posibilita la formación de ciudadanía (Lovón, 2019) y los procesos de aprendizaje intercultural. Este último toma la interpretación subjetiva de la realidad como punto de partida, donde la multiplicidad de la realidad no es reducible a una sola concreción, sino que representa una pluralidad y confrontación de conocimientos y patrones de interpretaciones. El aprendizaje intercultural ofrece el desarrollo de las habilidades de pensar y actuar socialmente. Sin embargo, debemos de reconocer los propios límites y la subjetividad que fortalecen la comprensión hacia los otros, aun cuando no coincidamos con imágenes congruentes de sus vidas.

\subsection{Oralidad y escritura}

Es sabido que todas las lenguas comienzan por la oralidad antes de poder llegar a la escritura, la cual depende de las características que tenga el 
desarrollo de la lengua. Hay culturas que aún no desarrollan sistemas de escritura, y también se sabe que no todas han desarrollado sistema de escritura silábica. Sin embargo, esto no indica que su sistema lingüístico sea deficiente o pobre, simplemente no han llegado a una etapa de abstracción gráfica para representar su lengua.

La escritura, a pesar de ser un elemento nuevo para las culturas indígenas del Perú, no deja de ser importante en el proceso comunicativo de estos pueblos, pues esta etapa visual de interacción comunicativa tiene como factor determinante el empleo de un medio (escrito) que no solo sirve como un simple medio de diálogo, sino que ayuda la comunicación en diferentes tiempos y espacios: un texto escrito lo podemos leer en cualquier momento y en cualquier locación; por el contrario, un discurso hablado no perdura en el tiempo y el espacio, a no ser que se registre de alguna forma. El habla sirve muy bien para efectos comunicativos in tempo, pero la escritura permite que la información se mantenga y transmita a otros medios receptores en diferentes latitudes. La enseñanza de este componente reviste una gran importancia.

Incentivar al niño a hablar en su lengua materna, por ejemplo, será uno de los primeros requisitos para reforzar esta habilidad y proyectarla hacia otras actividades como son la escritura y la lectura; más aún si las lenguas de nuestros alumnos tienen una mayor tradición oral que escrita. El aprendizaje, es decir, la búsqueda de la competencia comunicativa en el niño deberá iniciarse a través del incentivo de esta habilidad: el manejo y recreación oral de su lengua materna; solo así aprenderá a valorarla, a hacerla suya lo que, a la vez, le permitirá llevar a cabo un mejor acercamiento con otros individuos, así como con diferentes lenguas y culturas. (Cortez, 2000: 24)

La escritura como invento es muy valiosa y, por ello, es importante desarrollarla en la escuela como una de las habilidades lingüísticas que también permite interactuar con los demás y que apoyará el desarrollo de aprendizajes significativos en el niño (Cortez, 2000: 25). 
https://doi.org/10.46744/bapl.202001.006

\section{Metodología}

Este trabajo es descriptivo. La metodología consistió en la observación de dos sesiones de clases. Escogimos para el análisis una clase sobre plantas, porque fue el tema en común que apareció en ambas sesiones. La data que se presenta en esta investigación fue recogida en el año 2006. A pesar del tiempo, la información aún se mantiene vigente: las realidades no han cambiado según nuestras fuentes de contacto. El país aún mantiene las dinámicas educativas en asimetría.

\section{El estudio de caso}

\subsection{Descripción de la educación del shipibo-konibo de Canantipis- hca y del asháninka del Perené}

En la comunidad nativa de Canantipishca, a tres horas de Contamina, se encuentra el Centro Educativo 21060. Este centro solo tiene enseñanza en el nivel primario. La clase de shipibo se realiza en el curso de lengua nativa y, en este, el castellano es utilizado como recurso en alguna explicación metódica durante la clase o por falta de vocablo en la lengua local. Posteriormente, cuando termina la primaria, los niños van a escuelas mestizas donde solo se habla español y así terminan su educación escolar. En este centro hay un único profesor encargado de la enseñanza de la lengua. A la clase de L1 se le dedica cuatro horas a la semana.

En la comunidad nativa de Pucharini se encuentra el Centro Educativo Integrado Agroindustrial «Centro Piloto Pucharini», el cual cuenta con los niveles de primaria y secundaria, donde solo hay un maestro que fomenta la enseñanza y aprendizaje del asháninka del Perené. El asháninka es enseñado en cuarto de primaria y quinto de secundaria, con el fin de que puedan lograr su ingreso a las universidades e institutos con modalidad para indígenas. Es de advertir que en estas aulas hay alumnos de etnias yaneshas y quechua, ya que ellos cohabitan con la población asháninka en la misma comunidad. La escuela autoriza dos horas a la semana para la difusión y recuperación del asháninka de la zona. 


\subsection{La clase del día: La enseñanza sobre las plantas}

Una clase de shipibo consistió en la enseñanza y aprendizaje de las plantas y árboles, en la cual el docente del aula tenía que hacer conocer las diferencias que existe en la vegetación tanto de la zona como fuera de esta; el docente no utilizó los recursos del lugar, sino que se limitó a ejemplificaciones con material no muy pertinente para la clase, aunque llegase a realizar el objetivo trazado: enseñar acerca de las plantas. Él hizo una comparación con plantas de la zona andina para que los alumnos notaran la diferencia, pero siempre valorando lo autóctono o típico. De esta forma, se llegaba al conocimiento de una realidad distinta y se percibía un clima intercultural, aunque la interculturalidad se limitaba a la comparación y no tanto a la valoración.

Al margen de esto, la enseñanza y aprendizaje se realizaban con el empleo de la lengua indígena y se escuchaban algunas palabras en castellano. La clase era dictada por un profesor hablante de la lengua y el aula estaba provista de materiales didácticos tales como láminas con palabras en shipibo y motivos propios de la cultura. De la actividad realizada el día de la clase, pudimos observar que los niños hablaban por iniciativa propia, escuchaban a su maestro, respondían a sus preguntas y copiaban las palabras escritas que el profesor planteaba en la pizarra, en algunas ocasiones en castellano y en otras, en shipibo.

La clase de asháninka observada tenía como fin la enseñanza y el aprendizaje de las plantas, en especial de la palmera. La clase se realizó en dos espacios: dentro y fuera del aula, con una hora de duración en cada uno. El docente y los alumnos realizaron actividad de campo en el monte: el profesor les hablaba en castellano, les dictaba las partes de la palmera y ellos copiaban; luego, los niños trabajaron juntos en el recojo de material, pues se proyectaba realizar objetos con las partes de las palmeras recogidas, como abanicos y petates. En el salón de clases, el profesor hablaba en lengua asháninka. Esta parte consistió en nombrar y reconocer los objetos traídos del monte. Luego de que, el profesor escribía en la pizarra los nombres de los objetos en asháninka y las instrucciones en castellano, los niños se dedicaban a copiar. Después de esto, repetían 
https://doi.org/10.46744/bapl.202001.006

luego del maestro las palabras escritas. Se presentó la ocasión en que el maestro pidió señalar determinados objetos en asháninka a través de un juego de rapidez y atención. Asimismo, el profesor contrastó las plantas de la zona, en especial la palmera, con las de la costa, manifestando que todas tienen el mismo valor y las ventajas que pueden extraerse de cada una. Antes de esto, preguntó a los alumnos si conocían estas plantas y qué les habían contado sus padres sobre ellas. Los niños trataron de responder como podían.

\subsection{Comparación sobre la enseñanza y la interculturalidad}

En la siguiente tabla, se comparan las funciones comunicativas de las lenguas en la enseñanza escolar.

Tabla 1. Comparación sobre las funciones comunicativas en la enseñanza

\begin{tabular}{|l|l|l|}
\hline & Shipibo de Canantipishca & Asháninka del Perené \\
\hline $\begin{array}{l}\text { Transmisión } \\
\text { de conoci- } \\
\text { mientos por } \\
\text { parte del } \\
\text { maestro }\end{array}$ & $\begin{array}{l}\text { A través del shipibo en todo } \\
\text { momento. Muy pocas veces }\end{array}$ & $\begin{array}{l}\text { Dentro del aula: a } \\
\text { través del asháninka } \\
\text { en todo momento, } \\
\text { muy pocas veces en } \\
\text { castellano. }\end{array}$ \\
& & $\begin{array}{l}\text { Fuera del aula (en el } \\
\text { monte): en castellano. }\end{array}$ \\
\hline
\end{tabular}




\begin{tabular}{|c|c|c|}
\hline $\begin{array}{l}\text { Desarrollo } \\
\text { de enseñan- } \\
\text { za y apren- } \\
\text { dizaje en los } \\
\text { niños }\end{array}$ & $\begin{array}{l}\text { ORAL } \\
\text { Producción: hablan y respon- } \\
\text { den de por sí. } \\
\text { Comprensión: escuchan y } \\
\text { obedecen órdenes. } \\
\text { ESCRITURA } \\
\text { Producción: copian palabras } \\
\text { de la pizarra, estén en } \\
\text { shipibo o en castellano. } \\
\text { Lectura: leen y repiten } \\
\text { después del profesor. }\end{array}$ & $\begin{array}{l}\text { ORAL } \\
\text { Producción: repiten y } \\
\text { nombran cosas. } \\
\text { Comprensión: escu- } \\
\text { chan y obedecen órde- } \\
\text { nes a través del juego } \\
\text { didáctico. } \\
\text { ESCRITURA } \\
\text { Producción: copian lo } \\
\text { escrito en la pizarra o } \\
\text { lo dicho por el maestro } \\
\text { en asháninka o en } \\
\text { castellano. } \\
\text { Lectura: repiten las } \\
\text { palabras escritas que el } \\
\text { profesor menciona. }\end{array}$ \\
\hline $\begin{array}{l}\text { Oportunida- } \\
\text { des de usar } \\
\text { la lengua }\end{array}$ & $\begin{array}{l}\text { Los niños preguntan y } \\
\text { hablan por iniciativa propia. } \\
\text { Dentro del aula se dan ver- } \\
\text { daderas situaciones de uso. }\end{array}$ & Los niños observan. \\
\hline $\begin{array}{l}\text { Utilización } \\
\text { de materia- } \\
\text { les }\end{array}$ & $\begin{array}{l}\text { ORAL } \\
\text { Dibujos en láminas y en } \\
\text { la pizarra para comentar } \\
\text { (plantas de las zonas y de } \\
\text { lugares ajenos). } \\
\text { ESCRITURA } \\
\text { Uso de escritos en carteles (el } \\
\text { ambiente es letrado). }\end{array}$ & $\begin{array}{l}\text { ORAL } \\
\text { Plantas traídas del } \\
\text { monte y dibujos en la } \\
\text { pizarra para comentar } \\
\text { (plantas de la zona, las } \\
\text { ajenas son mencionadas } \\
\text { oralmente). } \\
\text { ESCRITURA } \\
\text { Uso de escritos en la } \\
\text { pizarra (el ambiente } \\
\text { es semiletrado, hay } \\
\text { escritos en castellano y } \\
\text { asháninka. }\end{array}$ \\
\hline
\end{tabular}


https://doi.org/10.46744/bapl.202001.006

En la siguiente tabla, se compara la concepción de interculturalidad que se maneja en la enseñanza escolar de las dos realidades.

Tabla 2. Comparación sobre el concepto de la interculturalidad

\begin{tabular}{|l|l|l|}
\hline & Shipibo de Canantipishca & Asháninka del Perené \\
\hline Identidad & $\begin{array}{l}\text { Reconoce la importancia } \\
\text { de las plantas del lugar y } \\
\text { se contrastan con las de la } \\
\text { sierra, dibujos en la pizarra } \\
\text { y lámina. Sin embargo, a } \\
\text { esta no se le da la misma } \\
\text { importancia. }\end{array}$ & $\begin{array}{l}\text { Reconoce la } \\
\text { importancia de las } \\
\text { plantas del lugar y se } \\
\text { contrastan con la de } \\
\text { la costa, mencionadas } \\
\text { oralmente. Sin } \\
\text { embargo, a esta no } \\
\text { se le da la misma } \\
\text { importancia. }\end{array}$ \\
\hline $\begin{array}{l}\text { Experiencias } \\
\text { previas }\end{array}$ & $\begin{array}{l}\text { El docente no trabaja con lo } \\
\text { que ya sabe el alumno. Los } \\
\text { conocimientos no se derivan } \\
\text { de la experiencia adquirida } \\
\text { tanto de lo propio como del } \\
\text { otro. }\end{array}$ & $\begin{array}{l}\text { El docente trata de } \\
\text { incorporar por lo que } \\
\text { saben de lo propio } \\
\text { y del otro. Trata de } \\
\text { preguntar qué les han } \\
\text { transmitido sus padres. }\end{array}$ \\
\hline $\begin{array}{l}\text { Trabajo de } \\
\text { campo }\end{array}$ & $\begin{array}{l}\text { Elumno en el proceso de } \\
\text { descubrir lo propio y lo } \\
\text { ajeno, la clase se desarrolla } \\
\text { dentro del aula. }\end{array}$ & $\begin{array}{l}\text { El docente propone } \\
\text { investigar. Trabaja un } \\
\text { momento de la clase } \\
\text { en el monte. Dirige } \\
\text { el trabajo hacia la } \\
\text { cooperación solidaria } \\
\text { y colaborativa en el } \\
\text { recojo de plantas, pero } \\
\text { se realiza en lengua } \\
\text { castellana. Asumen } \\
\text { la responsabilidad en } \\
\text { conjunto. }\end{array}$ \\
\hline
\end{tabular}


https://doi.org/10.46744/bapl.202001.006

\begin{tabular}{|c|c|c|}
\hline Práctica & $\begin{array}{l}\text { El concepto de intercultu- } \\
\text { ralidad se aboca al recono- } \\
\text { cimiento del otro y no a su } \\
\text { utilización. }\end{array}$ & $\begin{array}{l}\text { El concepto de inter- } \\
\text { culturalidad se aboca } \\
\text { al reconocimiento del } \\
\text { otro y a su utilización. } \\
\text { Se proyecta a elaborar } \\
\text { petates y alfombras } \\
\text { con las hojas de las } \\
\text { palmeras. }\end{array}$ \\
\hline $\begin{array}{l}\text { Valoración } \\
\text { de conoci- } \\
\text { mientos }\end{array}$ & $\begin{array}{l}\text { Valorizan lo suyo. Aceptan y } \\
\text { comprenden las diferencias } \\
\text { entre las variedades de } \\
\text { plantas, pero no les dan la } \\
\text { debida importancia. }\end{array}$ & $\begin{array}{l}\text { Valorizan lo suyo. } \\
\text { Aceptan y comprenden } \\
\text { las diferencias entre las } \\
\text { variedades de plantas. } \\
\text { El docente trata de } \\
\text { romper prejuicios de } \\
\text { superioridad. Reconoce } \\
\text { que el contacto entre } \\
\text { cultura es posible; } \\
\text { por tanto, provoca la } \\
\text { reflexión y ventajas. } \\
\text { Sin embargo, la } \\
\text { valoración del otro } \\
\text { se limita a lo hispano } \\
\text { y no a las etnias } \\
\text { yanesha y quechuas } \\
\text { que cohabitan en el } \\
\text { mismo entorno real. }\end{array}$ \\
\hline
\end{tabular}

4.4. Propuestas para la enseñanza de la lengua y la interculturalidad

Las siguientes propuestas están dirigidas para los tres últimos grados de primaria, ya que los niños poseen un grado de competencia oral y escrita más o menos compleja. 
https://doi.org/10.46744/bapl.202001.006

\subsubsection{Para los fines comunicativos}

Proponemos que para el shipibo de Canantipishca como L1 se debería enfatizar la escritura antes que la oralidad. Claro está que las propuestas para el desarrollo de los escritos deben partir de lo que se tiene, de los discursos hablados, y plasmarlos en el papel. En esta ocasión, tenemos dos propuestas para la posesión de la escritura. Estas pueden ser estrategias para la aplicación en cualquier tópico (acotaríamos que es bueno tratar de incorporar los contenidos de otras materias en el curso de Comunicación Integral o el curso de la lengua nativa):

1. El maestro pedirá que canten una canción tradicional, por ejemplo, la canción de la yuca, si el tema del día es las plantas; luego, pedirá que individualmente intenten escribir en sus cuadernos (para esto, el profesor ya habría enseñado a escribir algunas palabras en lengua indígena). Es bueno empezar con canciones cortas y que tengan palabras redundantes porque permitirá la fijación cognitiva de las palabras y de la estructura de frases. En ningún momento se pedirá la traducción de la canción.

Atsa s̈heati, atsa s̈heati

Kikin noe, kikin noe

Karibetan meskoa, karibetan meskoa

Kikin noe, kikin noe

2. El profesor contará un cuento y, antes de empezar, pedirá a los alumnos que traten de escribir las palabras que escuchan y que luego traten de sintetizar en un párrafo lo que han entendido. Estas tareas pueden trabajarse por separado, pues pensamos que sería lo más conveniente (hay que tener en cuenta el grado en que se encuentran los niños). Para la escritura del resumen, los niños pueden trabajar en grupo, a fin de discutir entre sí para saber lo que van a escribir y cómo lo van a hacer. A conti- 
nuación, presentamos un cuento que podría ser empleado por un profesor; también, se presenta el resumen que sería hecho por los niños como actividad de clase y el registro de algunas palabras repetidas que pudieran haber percibido.

\begin{tabular}{|c|c|}
\hline Cuento & Resumen \\
\hline $\begin{array}{l}\text { Moatianronki ika iki joni piti yamake- } \\
\text { tian mishki kaa kas̈honronki akai ki } \\
\text { icha yapa bikin, bikebinronki akai iki, } \\
\text { jakoma jonibaon bichinkin, jatian ja } \\
\text { joni onisshoko jan s̈hobonko kariba iki. } \\
\text { Jatianribi ika iki ö̈hen baken nashi } \\
\text { paketa, jatian oina iki joni neska akanai. } \\
\text { Jaskashon ö̈hen baken akai ki ja tita } \\
\text { os̈he yoikin, joni noibati piti bichinka- } \\
\text { na, jatian ja os̈he titan yoya iki, ja joni } \\
\text { akinon awe akin jan bake. } \\
\text { Jainshon ja ö̈hen yoya iki jan bake na- } \\
\text { man karibashon yoitawen ja joni bakish } \\
\text { ja jiwi mas aninko nenon jan bakebe- } \\
\text { tanbi oe koshira bea ishon. } \\
\text { Jaskashon ja baken ja joni neska akin } \\
\text { yoishon bakerano neriba iki jan titaiba. } \\
\text { Jaskatash ja joni yamekirishan netaiki ja } \\
\text { jiwi, mas ani ikain. Manatabironki ikai } \\
\text { oi beyamai, ja joniki moa pähkina. } \\
\text { Moa yametainbironki ikai, ja joni } \\
\text { jakomaboki sea tsamakana, itainbironki } \\
\text { ikai kikin oe Koshi bei. Ja oin mecha } \\
\text { aka ikai ja jakoma joniboki piiti. Jakon } \\
\text { jonin roNki ikai bochikishon oinkin } \\
\text { neskataintian. } \\
\text { Jaronki ika ika westiora castigo oshe ti- } \\
\text { tan bochikishon bema ja jakoma jonibo. }\end{array}$ & \begin{tabular}{l} 
Moatianronki ikai joni jawebi \\
piti yoma itan ikai yomeraikai, \\
moa piti bikebironki akanai \\
jakoma jonibon bichinti. Oshe \\
tita neskara oinshoronki akai \\
ja jakoma jonibo castiganki \\
pochinikon joni itan pochi- \\
nikon yapam jato imakin \\
Traducción: \\
Una vez un hombre que no \\
tenía nada que comer fue a \\
pescar, cuando regresaba a \\
su casa le quitan todo lo que \\
había pescado, la madre luna \\
observando todo esto manda \\
un castigo convirtiendo a to- \\
dos ellos en mitad pez y mitad \\
hombre. \\
\multicolumn{1}{c}{ Palabras repetidas } \\
Joni \\
Akai ki \\
Jains̈hon \\
Os̈he \\
Jaskähon \\
Bake \\
Akai
\end{tabular} \\
\hline
\end{tabular}


https://doi.org/10.46744/bapl.202001.006

\begin{tabular}{|l|l}
\hline Traducción: & Palabras repetidas: \\
Había una vez un hombre muy pobre y & Hombre \\
se fue al río a pescar, cuando fue pescó & Pescado \\
bastante, pero cuando se proponía ir a & Hizo \\
su casa unos hombres vienen y le quitan & En eso \\
todo lo que había pescado, y el hombre & Luna \\
regresó muy triste a su casa sin nada & Entonces \\
que comer. En ese tiempo que ocurría & Cuando \\
todo esto el hijo había bajado a bañarse & \\
y observa todo lo que había pasado. & \\
Cuando el hijo vuelve a subir le cuenta & \\
todo a su madre luna y ella escuchando & \\
la injusticia le dijo a su hijo que ayuda- & \\
ría al buen hombre. & \\
La madre luna le dijo a su hijo que & \\
bajare y le dijera al hombre que por la \\
mañana se subiera al árbol más grande \\
con su hijo, diciendo eso subió. \\
Al día siguiente el hombre con su \\
hijo hicieron lo que les había dicho el \\
muchacho. \\
El hombre cansado queriendo bajar del \\
árbol, ene so comienza a convertirse en \\
mitad pez y en mitad hombre. Y ese era \\
el castigo que la madre luna les había \\
enviado a los malos hombres. Mien- \\
tras que el hombre desde lo muy alto \\
observaba todo esto.
\end{tabular}

Para el caso del asháninka del Perené como L2, proponemos que debe enfatizarse más en la parte oral, darle un tratamiento especial. Puede dejarse la escritura de lado momentáneamente mientras esta no sea el soporte sobre el cual se apoye para recuperar el discurso hablado. Entonces, para el desarrollo de las habilidades comunicativas que posibilite el desarrollo oral de una segunda lengua, que debe ir paso a paso, planteamos lo siguiente: 
https://doi.org/10.46744/bapl.202001.006

1. El maestro deberá pedir objetos, tratar de dar órdenes que sean respondidas y entendidas, y preguntar por algunos elementos de su entorno relacionados con el tema de la clase, por ejemplo, los minerales. Estas órdenes y preguntas podrán haber sido escuchadas en su casa, por tanto, serán ejercitadas en el aula. O podrán relacionar lo que saben con el aprendizaje nuevo. Sería mejor si los niños lograsen comunicar un poco más de lo que se pide. Algunas preguntas y órdenes pueden ser las siguientes:

\begin{tabular}{|c|c|}
\hline $\begin{array}{c}\text { ¿Tsimatsi tsivi pibankotsi? } \\
\text { Apamakero tsivi }\end{array}$ & $\begin{array}{c}\text { ¿Hay sal en tu casa? } \\
\text { Tráeme la sal }\end{array}$ \\
\hline
\end{tabular}

2. Sugerimos que el maestro utilice los cuentos para el desarrollo de la habilidad verbal. El maestro podrá contar un cuento incompleto y los niños, en grupo o individualmente, cerrarán la historia. Es seguro que muchos de los niños habrán escuchado, por lo menos, algún cuento de la última generación de habla asháninka, los abuelos o los padres. En todo caso, el profesor dejará como actividad, antes de realizar la clase del día del cuento, que los niños pidan a sus familiares que les narren en lengua asháninka el cuento del tema solicitado. De esta manera, los niños tendrán la información requerida para actuar en la hora de clase de la lengua nativa.

\subsubsection{Para el tratamiento de la interculturalidad}

Proponemos lo siguiente para las dos realidades estudiadas:

1. En relación con las clases de shipibo de Canantipishca, sugerimos al maestro que el concepto de interculturalidad se operativice. Un ejemplo es llevar a los niños al monte a realizar investigación con el fin de reconocer lo propio (de acuerdo al tema propuesto para el día) y luego en el aula comparar las 
https://doi.org/10.46744/bapl.202001.006

utilidades que sus conocimientos poseen por reconocer, a la vez, la importancia de lo ajeno. A través de la práctica de la interculturalidad, se puede reconocer las ventajas que traen las diferencias de pensamiento y elementos materiales de otra cultura distinta de la zona. Este modelo sería, más o menos, el que se percibe para el caso del asháninka del Perené.

2. El caso del asháninka del Perené como L2 trata de incorporar la interculturalidad, pero hemos observado que en esta comunidad hay hablantes de otras lenguas como los yaneshas y quechuas. Por tanto, planteamos que se deben incorporar los saberes de estas culturas más cercanas al asháninka y ver cómo pueden ser de utilidad. Es posible encontrar en estas diferentes realidades como las formas de pesca. El papel del docente sería exponerlas en clase y hacer que todos los alumnos, de estas mismas etnias, llegasen a entenderse mutuamente cada vez más. Recordemos los consejos de Burga para los maestros EBI:

Para desarrollar una educación intercultural y pertinente, debes tratar de profundizar tu conocimiento sobre la herencia cultural de los niños y niñas con quienes trabajas y ampliar el que manejas sobre otros pueblos y grupos sociales existentes en el país. $(2003,14)$

\section{Conclusiones}

La situación de la enseñanza y aprendizaje con respecto a las funciones comunicativas en las horas de clase de la lengua nativa presentes en ambas realidades es diferente y, en general, en el Perú también lo es (Trapnell y Neira, 2004; López y Sichra, 2004).

Las estrategias de enseñanza y aprendizaje para la difusión del tema de las plantas en la lengua shipibo-konibo de Canantipishca están dirigidas a desarrollar las habilidades orales de comunicación y expresión, pero las escritas están limitadas al copiado y no a la escritura en sí. Pensamos que en esta comunidad, que tiene como L1 al shipibo, debería trabajarse en el 
https://doi.org/10.46744/bapl.202001.006

desarrollo de habilidades escritas, en la caracterización de su lengua en el papel, pues los niños dominan su lengua materna, pero no su escritura. Por otro lado, percibimos que no se trabaja la parte oral de los niños en la enseñanza del asháninka del Perené. Si lo que se quiere es recuperar la lengua indígena, las estrategias de enseñanza y aprendizaje deberían apuntar al desarrollo de los fines comunicativos; una lengua sobrevive si es hablada, $\mathrm{y}$ «retorna de la oscuridad» si es practicada. Mientras que la escritura es un componente poco trabajado en el caso del shipibo, consideramos que en el caso del asháninka del Perené debería trabajarse poco, pues la deuda se encuentra en el discurso hablado, en la palabra recuperada, a menos que se piense que la escritura llegue a constituirse como aliada plena para la revitalización de la oralidad asháninka del Perené. Asimismo, si lo escrito debe servir de soporte para lo oral, entonces los maestros deben procurar escribir en la lengua de la clase, la lengua nativa, y no mezclar el contexto escrito de una lengua con otra.

El desarrollo del enfoque intercultural en las horas de clase del tema de las plantas en el caso del shipibo-konibo de Canantipishca es casi nulo, se restringe a la comparación de plantas y no se valoriza las utilidades e importancia de lo otro, pues más se centran en la construcción y fortalecimiento de la identidad personal y cultural. Para la contrastación de variedades de plantas, utilizan materiales elaborados que contextualizan la comparación, pero que al parecer no brindan una interculturalidad natural, sino una interculturalidad «forzada» (si existe lo dicho), planteada para que se realice de tal o cual manera, sin salida y desarrollo libre de la misma reflexión. Es más, el enfoque intercultural parece ser desplazado por uno más monocultural, ya que el énfasis se da en lo propio. Mientras que en el caso del asháninka del Perené, lugar donde cohabitan no solo asháninkas, sino también yaneshas y quechuas, el desarrollo de la interculturalidad es tratada como un principio casi rector: se practica la valorización de lo propio y ajeno, la utilización de los saberes y la ventaja que traen las diferencias. Sin embargo, notamos que lo ajeno es valorizado en tanto pertenezca al mundo occidental, no se extraen los conocimientos de los grupos étnicos que cohabitan en el mismo entorno real. La cultura yanesha y quechua no son mencionadas en las horas de clase del tema de las plantas, a pesar de que el docente 
https://doi.org/10.46744/bapl.202001.006

sabe que los niños no solo son hijos de asháninkas del lugar. Además, suponemos que la operativización de la interculturalidad es débil.

Finalmente, cabe reconocer que las propuestas de EBI son distintas para cada zona. Las realidades sociolingüísticas deben llevar a los docentes a manejar las estrategias de enseñanza y aprendizaje oral y escrita de acuerdo con lo que piensan, pero deben percatarse de lo que se requiere realmente con urgencia en las situaciones de la lengua indígena como L1 o L2. Además, deben tener en cuenta que el tratamiento de la interculturalidad debe fortalecerse cada vez más si se busca vivir y convivir en un mundo más comprometido con el ser humano. 
https://doi.org/10.46744/bapl.202001.006

\section{BIBLIOGRAFÍA}

BURGA, E. (2003). Construyendo un mundo para todos. Guía para desarrollar la Interculturalidad en la escuela (III ciclo de educación primaria). Ministerio de Educación-DINEBI.

CORTEZ, M. (2000). Introducción a la Comunicación Integral. Perú: Ministerio de Educación, Programa FORTE-PE.

GALDAMES, V.; WALQUI, A. \& GUSTAFSON, B. (2005). Enseñanza de lengua indígena como lengua materna. La Paz: GTZ, INVENT.

GARCÍA, F. (1999). Fundamentos críticos de sociolingü̈ística. Madrid: Almería.

GODENZZI, J. (2001). Globalización, Multilingüismo y Educación. El caso del Perú (en línea). I Coloquio Tres Espacios Lingüísticos ante los Desafíos de la Mundialización. París, Francia. Recuperado de http://www.campues-oei.org/tres_espacios/icoloquio13.htm

HEISE, M. (2001). Interculturalidad. Creación de un concepto y desarrollo de una actitud. Lima: Programa FORTE-PE.

LÓPEZ, L. (1996). Donde el zapato aprieta: tendencias y desafíos de la educación en el Perú. Revista Andina, 12, 295-384.

LÓPEZ, L. E. y KÜPER, W. (2002). La educación intercultural bilingüe en América Latina: balance y perspectivas. Revista Iberoamericana de Educación, 20. Recuperado de https://doi.org/10.35362/ rie2001041

LÓPEZ, L. E. y SICHRA, I. (2004). La educación en áreas indígenas de América Latina: balances y perspectivas. Educación en la diversidad: experiencias y desafíos en la Educación Intercultural Bilingüe, pp. 121-149, Hernaiz, I. (coord.). Buenos Aires: Instituto Internacional de Planeamiento de la Educación (IIPE) y Unesco. 
https://doi.org/10.46744/bapl.202001.006

LOVÓN, M. (2019a). El «ciudadano» amazónico en el discurso político oficial. Lingüística y literatura, 75(40), pp. 38-61. Recuperado de http://doi.org/10.17533/udea.lyl.n75a02.

. (2016). Enseñanza de la lengua vernácula como segunda legua: Lenguas indígenas peruanas en estado de L2. Escritura y Pensamiento, 19(38), 185-210.

MINISTERIO DE EDUCACIÓN-DINEBI. (2002). Política Nacional de lenguas y culturas en la educación. Lima.

MORENO, F. (1998). Principios de sociolingüística y sociología del lenguaje. Barcelona: Ariel.

POZZI-ESCOT, I. (1998). El multilingüismo en el Perú. Cusco: CBC, PROEIB.

TRAPNELL, L. (2009). Entre lenguas, entre culturas: Proceso de construcción del programa FORMABIAP. Sistematización de una experiencia Perú. Guatemala: Instituto para el Desarrollo y la Innovación Educativa en Educación Bilingüe y Multicultural.

TRAPNELL, L., CALDERÓN, A., y FLORES, R. (2008). Interculturalidad, conocimiento y poder. Alcances de un proceso de investigación-acción en dos escuelas de la Amazonía peruana. Lima: Instituto del Bien Común, Ford Fundation, Oxfam América.

TRAPNELL, L. y NEIRA, E. (2004). Situación de la Educación Intercultural Bilingüe en el Perú. Lima: Banco Mundial, PROEIB, Andes.

VIGIL, N. (2004). El uso sistemático de las lenguas en las mal llamadas escuelas EBI del Perú. Lima.

. (2005). Las enseñanzas de lengua indígena como segunda lengua en la Amazonía peruana. Informe de consultoría. Lima. 
https://doi.org/10.46744/bapl.202001.006

WALQUI, A., \& Galdames, V. (2005). Enseñanza del castellano como segunda lengua. La Paz: GTZ, INVENT.

WALSH, C. (2000). Propuesta para el tratamiento de la Interculturalidad en la educación. Ministerio de Educación Inicial y Primaria-UNEBI.

ZÚÑIGA, M. (2008). La educación intercultural bilingüe. El caso peruano. Buenos Aires: Fundación Laboratorio de Políticas Públicas.

ZUÑIGA, M. y ANSIÓN, J. (1997). ¿Qué entender por interculturalidad? Lima: Foro Educativo.

. (2002). Repensando la educación bilingüe intercultural en el Perú: bases para una propuesta política. Lima: Red para el Desarrollo de las Ciencias Sociales. 